\title{
GOOD AND OK ULTRAFILTERS
}

\author{
BY
}

ALAN DOW

\begin{abstract}
In this paper we extend Kunen's construction of $\alpha^{+}$-good ultrafilters on $\mathscr{P}(\alpha)$ to more general algebras, as well as the construction of $\alpha^{+}$-OK ultrafilters. In so doing, we prove the existence of $\left(2^{\alpha} \times \alpha^{+}\right)$-independent matrices, as defined by Kunen, in these algebras. Some of the topological properties of the Stone spaces of these algebras are then investigated. We find points, for example, in $U(\alpha)$ which can be regarded as a generalization of weak $P$-points.
\end{abstract}

In 1964, Keisler introduced the notion of an $\alpha^{+}$-good ultrafilter on a field of sets (in particular, on $\mathscr{P}(\alpha)$ ). Keisler proved the existence of $\alpha^{+}$-good countably incomplete ultrafilters on $\mathscr{P}(\alpha)$ using the assumption that $2^{\alpha}=\alpha^{+}$. These ultrafilters are very important in model-theoretic applications. Kunen, in 1972, proved the existence of $\alpha^{+}$-good countably incomplete ultrafilters on $\mathscr{P}(\alpha)$ using the notion of families of large oscillation (introduced in [EK]) without any special set-theoretic assumptions. Shelah also used these ideas in his characterization of elementary equivalent structures. In 1978, Kunen weakened the notion of $\alpha^{+}$-good and introduced $c$-OK ultrafilters on $\mathscr{P}(\omega)$ modulo the finite sets. He constructed these ultrafilters using what he called a $(c \times c)$-independent matrix. These ultrafilters are very important in a topological setting because a $c$-OK (or even $\omega_{1}$-OK) ultrafilter on $\mathscr{P}(\omega) / \mathscr{P}_{\omega}(\omega)$ when viewed as a point in the space of free ultrafilters is a weak $P$-point. (A point $p \in X$ is a weak $P$-point if $p$ is not the limit point of any countable set.) These points and techniques have proved extremely useful for constructing special points and exhibiting nonhomogeneity in a large variety of spaces, most of it done by Jan van Mill. Kunen has asked about the existence of similar ultrafilters on $\mathscr{P}(\alpha) / \mathscr{P}_{\alpha}(\alpha)$ for larger cardinals $\alpha$. The main results of this paper are to show the existence of a $\left(2^{\alpha} \times \alpha^{+}\right)$-independent matrix on $\mathscr{P}(\alpha) / \mathscr{P}_{\alpha}(\alpha)$, the existence of $\alpha^{+}$-OK ultrafilters on $\mathscr{P}(\alpha) / \mathscr{P}_{\alpha}(\alpha)$ and some topological properties of these points. We also include some results on good ultrafilters, especially in regards to their existence in other boolean algebras such as the saturated boolean algebra extending earlier results of Negrepontis.

The author would like to thank Jan van Mill for helpful discussions and the referee for a generous and helpful report.

1. Preliminaries. Most of the notation and terminology we shall use is that of the Comfort and Negrepontis text [CN]. A small Greek letter shall usually refer to a

Received by the editors February 21, 1983 and, in revised form, June 18, 1984.

1980 Mathematics Subject Classification. Primary 06A40, 54D35.

Key words and phrases. Good ultrafilter, independent matrix, $U(\alpha)$.

(C)1985 American Mathematical Society $0002-9947 / 85 \$ 1.00+\$ .25$ per page 
cardinal, and a cardinal is an initial ordinal. When we refer to a cardinal $\alpha$ as a topological space it is assumed to have the discrete topology, and $\beta \alpha$ denotes the Cech-Stone compactification of $\alpha$. For a boolean algebra $B, S(B)$ refers to the Stone space of ultrafilters of $B$. For each $b \in B, \bar{b}=\{p \in S(B): b \in p\}$ is a closed and open subset (= clopen) of $S(B)$ and $\{\bar{b}: b \in B\}$ is a base for a compact Hausdorff topology on $S(B)$. For a set $X, \mathscr{P}(X)$ is the power set of $X$ and for a cardinal $\alpha, \mathscr{P}_{\alpha}(X)=\{Y \subset X:|Y|<\alpha\}$ and $\mathscr{P}^{\alpha}(X)=\{Y \subset X:|X-Y|<\alpha\}$. A filter $p$ on $\alpha$ is uniform if $|A|=\alpha$ for each $A \in p$. The subspace $U(\alpha)$ of $\beta \alpha$, consisting of the uniform ultrafilters on $\alpha$, is homeomorphic to $S\left(\mathscr{P}(\alpha) / \mathscr{P}_{\alpha}(\alpha)\right)$.

Let $f, g$ be functions from $\mathscr{P}_{\omega}(\alpha)$ into a boolean algebra $B$. Call $f$ monotone if $f(F) \leqslant f(G)$ whenever $F \supseteq G$ for $F, G \in \mathscr{P}_{\omega}(\alpha) ; f$ is multiplicative if $f(F) \wedge f(G)$ $=f(F \cup G)$ for $F, G \in \mathscr{P}_{\omega}(\alpha)$. We say that $f$ refines $g$, denoted $f \leqslant g$, if $f(F) \leqslant$ $g(F)$ for all $F \in \mathscr{P}_{\omega}(\alpha)$. We can now define an $\alpha$-good filter.

1.1. Definition. Let $B$ be a boolean algebra and let $\alpha$ be an infinite cardinal. A filter $\mathscr{F} \subset B$ is $\alpha$-good if, for each $\beta<\alpha$ and each monotone function $g: \mathscr{P}_{\omega}(\beta) \rightarrow$ $\mathscr{F}$, there is a multiplicative function $f$ from $\mathscr{P}_{\omega}(\beta)$ into $\mathscr{F}$ which refines $g$. $\mathscr{F}$ is said to be $\alpha-O K$ if, for each monotone function $g$ from $\mathscr{P}_{\omega}(\alpha)$ into $\mathscr{F}$ with $g(F)=g(G)$, whenever $|F|=|G|$, there is a multiplicative function $f$ from $\mathscr{P}_{\omega}(\alpha)$ into $\mathscr{F}$ refining $g$. (Note that an $\alpha^{+}$-good filter is $\alpha$-OK and, in both definitions, the assumption that $g$ be monotone is unnecessary.)

The notion $\alpha$-good is due to Keisler [Ke1] and $\alpha$-OK is due to Kunen [K2], although Keisler mentioned the notion of $\alpha-\mathrm{OK}$ in [Ke1] without giving it a name. A filter $\mathscr{F}$ on $\mathscr{P}(X)$ is called countably incomplete if there is a subset $\mathscr{G} \subset \mathscr{F}$, $|\mathscr{G}|=\omega$ and $\cap \mathscr{G}=\varnothing$. As mentioned above, Keisler showed that for a cardinal $\alpha$, with $2^{\alpha}=\alpha^{+}$, there are countably incomplete $\alpha^{+}$-good ultrafilters on $\alpha$. Kunen [K1] removed the assumption of $2^{\alpha}=\alpha^{+}$and later, Kunen [K2], showed the existence of $c$-OK ultrafilters of $\mathscr{P}(\omega) / \mathscr{P}_{\omega}(\omega)$ (i.e. on the boolean algebra of clopen subsets of $U(\omega)=\beta \omega-\omega)$. It is easy to see that there are no countably incomplete $\alpha^{++}$-good ultrafilters on $\alpha$ for any cardinal $\alpha$, however, we shall show that the situation for $\mathscr{P}(\alpha) / \mathscr{P}_{\alpha}(\alpha)$ is not the same.

Besides the boolean algebras $\mathscr{P}(\alpha) / \mathscr{P}_{\alpha}(\alpha)$ we shall be interested in the $\alpha$-saturated boolean algebras. We refer the reader to [CN, p. 277] for the definition of $\alpha$-saturated. We shall use the equivalence discovered by Negrepontis.

1.2. Definition. A boolean algebra $B$ is said to satisfy condition $H_{\alpha}$ if, given $A \cup C \subset B$ with $|A \cup C|<\alpha$, and, for each $A^{\prime} \in \mathscr{P}_{\omega}(A), C^{\prime} \in \mathscr{P}_{\omega}(C)$

$$
\bigvee\left(A^{\prime} \cup\{0\}\right)<\bigwedge\left(C^{\prime} \cup\{1\}\right)
$$

then there is a $b \in B$ satisfying $a<b<c$ for each $a \in A \cup\{0\}$ and $c \in C \cup\{1\}$.

1.3. TheOREm [N2, CN]. A boolean algebra $B$ is $\alpha$-saturated if and only if $B$ satisfies condition $H_{\alpha}$.

If $\alpha$ is a cardinal satisfying $\alpha=\alpha^{\alpha}=\Sigma\left\{\alpha^{\beta}: \beta<\alpha\right\}$, then there is a unique $\alpha$-saturated boolean algebra of cardinality $\alpha$. However, for any cardinal $\alpha$, there are $\alpha^{+}$-saturated boolean algebras of cardinality $2^{\alpha}$. Indeed, $\mathscr{P}(\omega) / \mathscr{P}_{\omega}(\omega)$ is $\omega_{1}$-saturated, and if $p$ is an $\alpha^{+}$-good ultrafilter on $\alpha$, then the reduced ultrapower 
$\left[\mathscr{P}(\omega) / \mathscr{P}_{\omega}(\omega)\right]^{\alpha} / p$ is $\alpha^{+}$-saturated (see [Ke1]). There are two conditions which are weaker than $H_{\alpha}$ which are also satisfied by $\mathscr{P}(\alpha) / \mathscr{P}_{\alpha}(\alpha)$ and which are sufficient to enable us to construct $\alpha^{+}-$OK points for regular $\alpha$. We shall say that a boolean algebra $B$ satisfies condition $F_{\alpha}$ if, for any $A \cup C \subset B$ with $|A \cup C|<\alpha$ and $a \wedge c=0$ for each $a \in A$ and $c \in C$, there is a $b \in B$ with $a \leqslant b$ for each $a \in A$ and $b \wedge c=0$ for each $c \in C$. (The essential difference between $F_{\alpha}$ and $H_{\alpha}$ is that $b$ need not be strictly greater than the elements of $A$.) We have called this condition $F_{\alpha}$ because it is equivalent to the condition that $S(B)$ is an $F_{\alpha}$-space [CN]. A subset $A$ of a boolean algebra $B$ is called cellular if, for each $a, c \in A, a \neq c$ implies $a \wedge c=0$. We shall say that $B$ satisfies condition $E_{\alpha}$ if, for each $b \in B \backslash\{0\}$, there is a cellular $A \subset(b]=\{c \in B: c \leqslant b\}$ with $|A|=\alpha$ and, for any such $A$, there is a $c \in(b] \backslash\{0\}$ such that $A \cup\{c\}$ is cellular. We end this section with a result which assures that the results of the remainder of the paper apply to $\mathscr{P}(\alpha) / \mathscr{P}_{\alpha}(\alpha)$ and $U(\alpha)$.

1.4. THEOREM [N1, CN]. Let $\alpha$ be an infinite cardinal and let $\lambda=\operatorname{cf}(\alpha)$ (cofinality of $\alpha)$. The boolean algebra $\mathscr{P}(\alpha) / \mathscr{P}_{\alpha}(\alpha)$ satisfies the conditions $F_{\lambda^{+}}$and $E_{\lambda}$.

2. Independent matrices. As mentioned earlier, Kunen used families of large oscillation to construct $\alpha^{+}$-good ultrafilters on $\mathscr{P}(\alpha)$ and $(c \times c)$-independent matrices to construct $c$-OK ultrafilters on $\mathscr{P}(\omega) / \mathscr{P}_{\omega}(\omega)$. In this section we shall construct analogous independent matrices which will facilitate the construction of $\alpha^{+}$-OK ultrafilters on $\mathscr{P}(\alpha) / \mathscr{P}_{\alpha}(\alpha)$. We will also construct families which will allow us to construct ultrafilters which are simultaneously $\alpha^{+}$-good and $\alpha^{+}$-OK (recall that an $\alpha^{+}$-good filter is $\alpha$-OK). We begin by defining the notions of large oscillation and independent matrices.

2.1. Definition. Let $\alpha$ be a cardinal, $\mathscr{F}$ a filter on $\mathscr{P}(\alpha)$ and $\mathscr{S} \subset \alpha^{\alpha}$. Then $\mathscr{S}$ is of large oscillation mod $\mathscr{F}$ if, for any $n \in \omega,\left\{\zeta_{i}: i<n\right\} \subset \alpha,\left\{h_{i}: i<n\right\}$ distinct elements of $\mathscr{S}$, and $A \in \mathscr{F}$, we have $\left\{\xi<\alpha: h_{i}(\xi)=\zeta_{i}\right.$ for $\left.i<n\right\} \cap A \neq \varnothing$.

2.2. Definition. Let $\alpha, \beta$ be cardinals, $B$ a boolean algebra, $\mathscr{F}$ a filter of $B$ and $\left\{b_{\gamma \delta}: \gamma<\alpha, \delta<\beta\right\} \subset B$. The matrix $\left\{b_{\gamma, \delta}: \gamma<\alpha, \delta<\beta\right\}$ is an $(\alpha \times \beta)$-independent matrix $\bmod \mathscr{F}$ if, for each $\gamma<\alpha$ and $\delta<\xi<\beta, b_{\gamma, \delta} \wedge b_{\gamma, \xi}=0$ and, for each finite $F \subset \alpha$, function $f \in \beta^{F}$ and $a \in \mathscr{F}, \wedge\left\{b_{\gamma, f(\gamma)}: \gamma \in F\right\} \wedge a \neq 0$. If $\mathscr{F}=\varnothing$, then we shall call the above matrix an $(\alpha \times \beta)$-independent matrix.

In order to construct $\alpha^{+}$-OK ultrafilters of $\mathscr{P}(\alpha) / \mathscr{P}_{\alpha}(\alpha)$ we will need a $\left(2^{\alpha} \times \alpha^{+}\right)$independent matrix. For this we need two preliminary results. We shall omit the proof of the first but one can be found in $[\mathbf{C N}]$ as well as [EK].

2.3. LEMMA [EK]. For each infinite $\alpha$, there is a set $\mathscr{S} \subset \alpha^{\alpha}$ of large oscillation $\bmod \mathscr{P}^{\alpha}(\alpha)$ with $|\mathscr{S}|=2^{\alpha}$.

The next result is new but is similar in spirit to a construction in [DvM].

2.4. ThEOREM. Let $B$ be a boolean algebra which satisfies conditions $F_{\alpha^{+}}$and $E_{\alpha}$. Then $B$ contains an $\left(\alpha^{+} \times \alpha^{+}\right)$-independent matrix.

Proof. Fix a bijection $f: \alpha^{+} \rightarrow \alpha^{+} \times \alpha^{+}$and for $\gamma \in \alpha^{+}$let $r(\gamma)=\{\xi\}$ where $f(\gamma) \in\{\xi\} \times \alpha^{+}$. We shall define our matrix $\left\{a_{\gamma, \delta}:(\gamma, \delta) \in \alpha^{+} \times \alpha^{+}\right\}$by inducting 
along the indices with the order imposed by $f$. At the same time we shall define elements of $B, b_{F}$ for $F \in \mathscr{P}_{\omega}\left(\alpha^{+}\right)$, whose pupose will be to keep track of the meets of the $a_{\gamma, \delta}$ 's. We will also be defining subsets of $B, H(F)$ for $F \in \mathscr{P}_{\omega}\left(\alpha^{+}\right)$, whose purposes are to overcome the obviously minor difficulty imposed by the fact that $B$ does not necessarily satisfy $H_{\alpha^{+}}$(as does $\left.\mathscr{P}(\omega) / \mathscr{P}_{\omega}(\omega)\right)$.

We begin the induction by letting $b_{\varnothing}=1$ and let $H(\varnothing)$ be an arbitrary cellular family of $B$ of size $\alpha$ which exists since $B$ satisfies $E_{\alpha}$. Choose $b_{\{0\}} \in B$ such that $H(\varnothing) \cup\left\{b_{\{0\}}\right\}$ is cellular which we may do by $E_{\alpha}$. Now choose $H(\{0\}) \subset\left(b_{\{0\}}\right]$, a cellular family of size $\alpha$. Define $a_{f(0)}=b_{\{0\}}$, and suppose that $\gamma<\alpha^{+}$and that we have defined $a_{f(\xi)}$ for $\xi<\gamma, b_{F}$ and $H(F)$ for $F \in \mathscr{P}_{\omega}(\gamma)$ so that:

(i) $b_{F} \leqslant b_{G}$ if $F \supset G$ and $\max G<\xi$ for $\xi \in F \backslash G$ for $F, G \in \mathscr{P}_{\omega}(\gamma)$;

(ii) $H(F) \subset\left(b_{F}\right]$ is a cellular family of size $\alpha$ for $F \in \mathscr{P}_{\omega}(\gamma)$ with $|r[F]|=|F|$;

(iii) $\left\{b_{F \cup\{\xi\}}\right\} \cup H(F)$ is cellular for $F \cup\{\xi\} \in \mathscr{P}_{\omega}(\gamma)$ with $\max F<\xi$ and $|r[F \cup\{\xi\}]=| F \cup\{\xi\} \mid$;

(iv) $\left\{a_{f(\xi)}\right\} \cup H(F)$ is cellular if $\xi \notin F$ for $F \cup\{\xi\} \in \mathscr{P}_{\omega}(\gamma)$;

(v) $0<b_{F \cup\{\xi\}}<a_{f(\xi)}$ if $\max F<\xi$ and $|r[F \cup\{\xi\}]|=|F \cup\{\xi\}|$ for $F \cup\{\xi\}$ $\in \mathscr{P}_{\omega}(\gamma)$

(vi) $a_{f(\xi)} \wedge a_{f(\delta)}=0$ if $r(\xi)=r(\delta)$ for $\xi<\delta<\gamma$.

We define $a_{f(\gamma)}, b_{F \cup\{\gamma\}}$ and $H(F \cup\{\gamma\})$ for each $F \in \mathscr{P}_{\omega}(\gamma)$. For $F \in \mathscr{P}_{\omega}(\gamma)$ with $|r[F \cup\{\gamma\}]|<|F \cup\{\gamma\}|$, let $b_{F \cup\{\gamma\}}=0$ and $H(F \cup\{\gamma\})=\varnothing$. (The idea here is that if $\xi<\gamma$ and $r(\xi)=r(\gamma)$ then $a_{f(\xi)} \wedge a_{f(\gamma)}=0$; hence $b_{\{\xi, \gamma\}}=0$.) Now, let $F \in \mathscr{P}_{\omega}(\gamma)$ with $|r[F \cup\{\gamma\}]|=|F|+1$. Note that for $\xi \in \gamma \backslash F,\left\{a_{f(\xi)}\right\} \cup H(F)$ is cellular by (iv). Since $B$ satisfies $F_{\alpha^{+}}$, there is a $b^{\prime} \in B$ satisfying $\left\{b^{\prime}\right\} \cup H(F)$ is cellular and $a_{f(\xi)} \leqslant b^{\prime}$ for $\xi \in \gamma \backslash F$. Also, we can choose $b_{F \cup\{\gamma\}} \in\left(b_{F}\right]$ such that $\left\{b_{F \cup\{\gamma\}}\right\} \cup H(F) \cup\left\{b^{\prime}\right\}$ is cellular by $E_{\alpha}$. Now choose $H(F \cup\{\gamma\}) \subset\left(b_{F \cup\{\gamma\}}\right]$ as in (ii). Clearly condition (i)-(iv) are satisfied. Now we define $a_{f(\gamma)}$. Let $A=$ $\left\{b_{F \cup\{\gamma\}}: F \in \mathscr{P}_{\omega}(\gamma)\right.$ and $\left.r(\gamma) \notin r[F]\right\}$ and

$$
C=\left\{a_{f(\xi)}: \xi<\gamma \text { and } r(\xi)=r(\gamma)\right\} \cup \cup\left\{H(F): F \in \mathscr{P}_{\omega}(\gamma)\right\} ;
$$

$|A \cup C|<\alpha^{+}$. For $a \in A$ and $c \in C$, it follows that $a \wedge c=0$ by (iii) if $c \in H(F)$ for some $F \in \mathscr{P}_{\omega}(\gamma)$ and, by the definition of $b_{F \cup\{\gamma\}}$ for $F \in \mathscr{P}_{\omega}(\gamma)$ if $c=a_{f(\xi)}$ for $\xi<\gamma$. Hence we may choose $a_{f(\gamma)} \in B$ so that $a \leqslant a_{f(\gamma)}$ and $a_{f(\gamma)} \wedge c=0$ for $a \in A, c \in C$ since $B$ satisfies $F_{\alpha}{ }^{.}$. Clearly (i)-(vi) are satisfied. This completes the induction.

Let us check that $\left\{a_{\gamma, \delta}:(\gamma, \delta) \in \alpha^{+} \times \alpha^{+}\right\}$is an independent matrix. First we let $\gamma \in \alpha^{+}$and $\delta<\xi<\alpha^{+}$and check that $a_{\gamma, \delta} \wedge a_{\gamma, \xi}=0$. By the definition of $r$, $r\left(f^{\leftarrow}((\gamma, \delta))\right)=\{\gamma\}=r\left(f^{\leftarrow}((\gamma, \xi))\right)$. Therefore by condition (vi), $a_{\gamma, \delta} \wedge a_{\gamma, \xi}=0$. Now let $G \in \mathscr{P}_{\omega}\left(\alpha^{+}\right)$and $g \in\left(\alpha^{+}\right)^{G}$. Let $F=\left\{f^{\leftarrow}(\gamma, g(\gamma)): \gamma \in G\right\} \in \mathscr{P}_{\omega}\left(\alpha^{+}\right)$ and note that $|F|=|r[F]|$. Clearly $\wedge\left\{a_{\gamma, g(\gamma)}: \gamma \in G\right\}=\wedge\left\{a_{f(\xi)}: \xi \in F\right\}$. Let $\xi=\max F$ and note that by (iii), $b_{F}=b_{F \backslash\{\xi\} \cup\{\xi\}}>0$. Therefore, by (v), $0<b_{F}<$ $a_{f(\xi)}$ for $\xi \in F$, and we are done.

Now we combine the previous two results following an idea of [BF].

2.5. TheOREM. Let $B$ be a boolean algebra satisfying $F_{\alpha^{+}}$and $E_{\alpha^{*}}$. Then $B$ has a $\left(2^{\alpha} \times \alpha^{+}\right)$-independent matrix. 
Proof. Since $B$ satisfies $E_{\alpha}$, we may choose a cellular family $\left\{b_{\gamma}: \gamma \in \alpha\right\} \subset B$. Now it is easily seen that each boolean algebra $\left(b_{\gamma}\right], \gamma \in \alpha$, also satisfies $F_{\alpha^{+}}$and $E_{\alpha}$. Therefore we may choose, for each $\gamma \in \alpha$, and $\left(\alpha \times \alpha^{+}\right)$-independent matrix $\left\{a(\xi, \delta, \gamma): \xi \in \alpha, \delta \in \alpha^{+}\right\}$so that $a(\xi, \delta, \gamma)<b_{\gamma}$ for each $(\xi, \delta) \in \alpha \times \alpha^{+}$, by Theorem 2.4. Now, from Lemma 2.3 , choose a family $\mathscr{S} \subset \alpha^{\alpha}$ of large oscillation $\bmod \mathscr{P}^{\alpha}(\alpha)$ with $|\mathscr{S}|=2^{\alpha}$. We shall define an $\left(\mathscr{S} \times \alpha^{+}\right)$-independent matrix on $B$. For each $f \in \mathscr{S}$, we perform the following induction.

Let $f \in \mathscr{S}$. Suppose that $\beta<\alpha^{+}$and, for $\delta<\beta$, that we have defined $A_{f, \delta} \in B$ so that, (i) for $\gamma \in \alpha, A_{f, \delta} \wedge b_{\gamma}=a(f(\gamma), \delta, \gamma)$ and (ii) $\gamma_{1}<\gamma_{2}<\beta$ implies $A_{f, \gamma_{1}} \wedge$ $A_{f, \gamma_{2}}=0$.

Let us define $A_{f, \beta}$. We first observe that, for $\xi \in \alpha$ and $\delta<\beta, a(f(\xi), \beta, \xi) \wedge$ $A_{f, \delta}=0$ since $A_{f, \delta} \wedge b_{\xi}=a(f(\xi), \delta, \xi)$ and $a(f(\xi), \beta, \xi) \wedge a(f(\xi), \delta, \xi)=0$. Since $B$ satisifies condition $F_{\alpha^{+}}$we can choose $A_{f, \beta} \in B$ so that $a(f(\xi), \beta, \xi)<A_{f, \beta}$ for $\xi \in \alpha, A_{f, \beta} \wedge A_{f, \delta}=0$ for $\delta<\beta$, and $A_{f, \beta} \wedge b_{\xi}-a(f(\xi), \beta, \xi)=0$ for $\xi \in \alpha$. This completes the induction.

Let us check that $\left\{A_{f, \delta}: f \in \mathscr{S}, \delta \in \alpha^{+}\right\}$is an independent matrix. Clearly, by the construction, for each $f \in \mathscr{S}$ and $\delta_{1}<\delta_{2}<\alpha^{+}, A_{f, \delta_{1}} \wedge A_{f, \delta_{2}}=0$. Let $\left\{f_{i}: i \in n\right\}$ $\subset \mathscr{S}$ be distinct and let $g \in\left(\alpha^{+}\right)^{n}$; it suffices to show that

$$
\bigwedge_{i<n} A_{f_{i}, g(i)} \neq 0
$$

Since $\mathscr{S}$ is of large oscillation we may choose any $\xi \in \alpha$ with $f_{i}(\xi)=i$ for $i \in n$. It follows that

$$
\bigwedge_{i<n} A_{f_{i}, g(i)}>\bigwedge_{i<n} a\left(f_{i}(\xi), g(i), \xi\right)
$$

Now $\wedge\left\{a\left(f_{i}(\xi), g(i), \xi\right): i \in n\right\} \neq 0$ since $\left\{a(\gamma, \delta, \xi): \gamma \in \alpha, \delta \in \alpha^{+}\right\}$is an independent matrix.

2.6. Corollary. For each infinite regular cardinal $\alpha$, there is a $\left(2^{\alpha} \times \alpha^{+}\right)$independent matrix on $\mathscr{P}(\alpha) / \mathscr{P}_{\alpha}(\alpha)$. (Equivalently, there is a $\left(2^{\alpha} \times \alpha^{+}\right)$-independent matrix $\bmod \mathscr{P}^{\alpha}(\alpha)$ on $\mathscr{P}(\alpha)$.)

We combine the notions of large oscillation and independent matrix in the following

2.7. Definition. For a boolean algebra $B$, a cellular family $\left\{b_{\gamma}: \gamma \in \alpha\right\} \subset B$, $\mathscr{T} \subset \alpha^{\alpha}$ a filter $\mathscr{F}$ of $B$, and a matrix $\left\{A_{\beta, \delta}: \beta \in \mathscr{S}, \delta \in \alpha^{+}\right\}$, we say that $\left\langle\left\{A_{\beta, \delta}\right.\right.$ : $\left.\left.\beta \in \mathscr{S}, \delta \in \alpha^{+}\right\}, \mathscr{T}\right\rangle$ is $\left\{b_{\gamma}: \gamma \in \alpha\right\}$-independent $\bmod \mathscr{F}$ if $A_{\beta, \delta} \wedge A_{\beta, \delta^{\prime}}=0$, for $\delta<\delta^{\prime}<\alpha^{+}$and $\beta \in \mathscr{S}$, and, for each $F \in \mathscr{P}_{\omega}(\mathscr{S}), G \in \mathscr{P}_{\omega}(\mathscr{T})$, and functions $s \in\left(\alpha^{+}\right)^{F}, t \in \alpha^{G}$ and $b \in \mathscr{F},\left\{\gamma \in \alpha: g(\gamma)=t(g)\right.$ for $g \in G$ and $b \wedge b_{\gamma} \wedge$ $\left.\wedge\left\{A_{\beta, s(\beta)}: \beta \in F\right\} \neq 0\right\} \neq \varnothing$.

Then we have the next result as a corollary to the proof of Theorem 2.5.

2.8. THEOREM. Let $B$ be a boolean algebra satisfying $F_{\alpha^{+}}$and $E_{\alpha}$. Also let $\mathscr{S}$ and $\mathscr{T}$ be disjoint subsets of $\alpha^{\alpha}$ so that $\mathscr{S} \cup \mathscr{T}$ is of large oscillation $\bmod \mathscr{P}^{\alpha}(\alpha)$ and let $\left\{b_{\gamma}\right.$ : $\gamma \in \alpha\}$ be a cellular family of $B$. Define $\mathscr{F}$ to be the filter of $B$ consisting of $\{b \in B$ : $\left.\left\{\gamma \in \alpha: \quad b_{\gamma}<b\right\} \in \mathscr{P}^{\alpha}(\alpha)\right\}$. Then there is a $\left(|\mathscr{S}| \times \alpha^{+}\right)$-matrix $\left\{A_{f, \delta}: f \in \mathscr{S}\right.$, 
$\left.\delta \in \alpha^{+}\right\} \subset B$ such that $\left\langle\left\{A_{f, \delta}: f \in \mathscr{S}, \delta \in \alpha^{+}\right\}, \mathscr{T}\right\rangle$ is $\left\{b_{\gamma}: \gamma \in \alpha\right\}$-independent $\bmod \mathscr{F}$.

Proof. As in the proof of 2.5 , construct $\left\{A_{f, \delta}: f \in \mathscr{S}, \delta \in \alpha^{+}\right\}$. Note that if $F \in \mathscr{P}_{\omega}(\mathscr{S})$ and $\gamma \in \alpha$ is such that $|\{f(\gamma): f \in F\}|=|F|$, then

$$
b_{\gamma} \wedge \wedge\left\{A_{f, s(f)}: f \in F\right\} \neq 0
$$

for any $s \in\left(\alpha^{+}\right)^{F}$. Now let $F, G, s, t$ and $b$ be as in 2.7. Choose arbitrarily distinct $\zeta_{f} \in \alpha \backslash\{t(g): g \in G\}$ for $f \in F$. Since $S \cup \mathscr{T}$ is of large oscillation $\bmod \mathscr{P}^{\alpha}(\alpha)$ and $\left\{\gamma \in \alpha: b_{\gamma}<b\right\} \in \mathscr{P}^{\alpha}(\alpha),\left\{\gamma \in \alpha: g(\gamma)=t(g)\right.$ and $f(\gamma)=\zeta_{f}$ for $g \in G$ and $f \in F\} \cap\left\{\gamma \in \alpha: b_{\gamma}<b\right\} \neq \varnothing$. Thus it follows that

$$
\left\{\gamma \in \alpha: g(\gamma)=t(g) \text { for } g \in G, b \wedge b_{\gamma} \wedge \wedge\left\{A_{f \zeta_{f}}: f \in F\right\} \neq 0\right\} \neq \varnothing .
$$

2.9. REMARK. It is certainly consistent that there is no $\left(2^{\alpha} \times 2^{\alpha}\right)$-independent matrix on $\mathscr{P}(\alpha) / \mathscr{P}_{\alpha}(\alpha)$ for $\alpha>\omega$; since it is consistent there is not even a cellular family of size $2^{\alpha}$. However, we do not know if there is always such a matrix on an $\alpha^{+}$-saturated boolean algebra as is the case for $\mathscr{P}(\omega) / \mathscr{P}_{\omega}(\omega)$. It is also worth mentioning that the construction of such a matrix on $\mathscr{P}(\omega) / \mathscr{P}_{\omega}(\omega)$ is done by directly defining subsets of $\omega$ but no such construction is known for larger $\alpha$ (except for $\alpha$ a strong limit).

3. Constructing good and OK ultrafilters. In this section we carry out the principle constructions, beginning with 3.2 which is a slight generalization of Kunen's construction of $\alpha^{+}$-good ultrafilters on $\alpha$. It also generalizes Negrepontis' [N3] construction of such ultrafilters on $\alpha^{+}$-saturated algebras by removing the cardinality assumption. Next we construct $\alpha^{+}-\mathrm{OK}$ ultrafilters, generalizing Kunen's construction [K2] of $c$-OK ultrafilters of $\mathscr{P}(\omega) / \mathscr{P}_{\omega}(\omega)$. Finally in 3.5 , we combine the two constructions. First we state the following

3.1. Definition. Let $B$ be a boolean algebra and let $\eta$ be a cardinal. A filter $\mathscr{F}$ $\subset B$ is called $\eta$-uniform $\left((\eta, \omega)\right.$-uniform) if there is a cellular family $\left\{c_{\gamma}: \gamma \in \eta\right\} \subset B$ such that, for each $b \in \mathscr{F},\left|\left\{\gamma \in \eta: b_{\gamma} \wedge b \neq 0\right\}\right|=\eta$ (if, in addition, there is a countable $C \subset \mathscr{F}$ such that, for each $\gamma \in \eta$, there is a $c \in C$ with $\left.c \wedge b_{\gamma}=0\right)$. $\mathscr{F}$ is an $(\omega, \omega)$-uniform ultrafilter if and only if $\mathscr{F}$ is not a $P$-point in $S(B)$.

Throughout the remainder of this section, fix an infinite cardinal $\alpha$ and a boolean algebra $B$ of cardinality $2^{\alpha}$ which satisfies conditions $F_{\alpha^{+}}$and $E_{\alpha}$. Recall that any $\alpha^{+}$-saturated algebra of cardinality $2^{\alpha}$ is such an algebra and that if $\alpha$ is regular, then so is $\mathscr{P}(\alpha) / \mathscr{P}_{\alpha}(\alpha)$. Let $\left\{b_{\gamma}: \gamma \in \alpha\right\} \subset B$ be cellular and let $\mathscr{S} \cup \mathscr{T} \subset \alpha^{\alpha}$ be disjoint, $|\mathscr{S}|=|\mathscr{T}|=2^{\alpha}$ such that $\mathscr{S} \cup \mathscr{T}$ is of large oscillation $\bmod \mathscr{P}^{\alpha}(\alpha)$.

Fix, from Theorem 2.8, a matrix $\left\{A_{f, \beta}: f \in \mathscr{S}, \beta \in \alpha^{+}\right\}$so that $\left\langle\left\{A_{f, \beta}: f \in \mathscr{S}\right.\right.$, $\left.\left.\beta \in \alpha^{+}\right\}, \mathscr{T}\right\rangle$ is $\left\{b_{\gamma}: \gamma \in \alpha\right\}$-independent $\bmod \mathscr{P}^{\alpha}(\alpha)$ (see Definition 2.7).

3.2. TheOREM. For any infinite cardinal $\eta \leqslant \alpha$ there is an $(\eta, \omega)$-uniform $\alpha^{+}$-good ultrafilter on $B$.

Proof. Let $\mathscr{D}$ be the set which is the union of $B$ with $\{S: S$ is a monotone map from $\mathscr{P}_{\omega}(\alpha)$ into $\left.B\right\}$. Since $|\mathscr{D}|=2^{\alpha}$ we may choose an indexing $\left\{d_{\xi}: \xi<2^{\alpha}\right\}$ so that each monotone function appears $2^{\alpha}$ times. We will construct by induction of length $2^{\alpha}$ an ultrafilter on $B$. First we will make sure that this filter will be 
$(\eta, \omega)$-uniform. Let $h \in \mathscr{T}$ be arbitrary. Since $B$ satisifies $F_{\alpha^{+}}$we may define by induction a cellular family $\left\{c_{\gamma}: \gamma<\eta\right\} \subset B$ such that, for each $\gamma<\eta,\{\xi \in \alpha$ : $\left.b_{\xi} \wedge c_{\gamma} \neq 0\right\}=\left\{\xi \in \alpha: b_{\xi}<c_{\gamma}\right\}=h^{\leftarrow}(\gamma)$.

Again, because $B$ satisfies $F_{\alpha^{+}}$, we may choose, for $\gamma<\eta$ and $k \in \omega$, an element $c_{\gamma, k}$ satisfying $\left\{\xi \in \eta: c_{\xi} \wedge c_{\gamma, k} \neq 0\right\}=\left\{\xi \in \eta: c_{\xi}<c_{\gamma, k}\right\}=\{\xi \in \eta: \xi=\lambda+m$ for some limit ordinal $\lambda$ and integer $m>k\}$. Let $\mathscr{F}_{0}$ be the filter generated by $\left\{c_{\gamma, k}: \gamma \in \eta, k \in \omega\right\} \cup\left\{b \in B: b_{\gamma}<b\right.$ for all $\left.\gamma<\alpha\right\}$. Observe that any filter containing $\mathscr{F}_{0}$ will be $(\eta, \omega)$-uniform as exhibited by the cellular family $\left\{c_{\gamma}\right.$ : $\gamma \in \eta\}$. Indeed, if $b \in B$ is such that $\left|\left\{\gamma \in \eta: b \wedge c_{\gamma} \neq 0\right\}\right|<\eta$, then there is a $\gamma<\eta$ such that $b \wedge c_{\gamma, 0}=0$. However, this implies that $1-b \in \mathscr{F}_{0}$ since $1-b>$ $c_{\gamma, 0}$. It is easy to see that $\langle\varnothing, \mathscr{T} \backslash\{h\}\rangle$ (or even $\left\langle\left\{A_{f, \beta}: f \in \mathscr{S}, \beta \in \alpha^{+}\right\}\right.$, $\mathscr{T} \backslash\{h\}\rangle)$ is $\left\{b_{\gamma}: \gamma \in \alpha\right\}$-independent $\bmod \mathscr{F}_{0}$ since, for each $b \in \mathscr{F}_{0}$ and $\xi \in \alpha$, there is a $\xi^{\prime}<\alpha$ with $\xi<\xi^{\prime}$ such that $b_{\gamma}<b$ for each $\gamma \in h\left(\xi^{\prime}\right)$. Let $\mathscr{T}_{0}=\mathscr{T} \backslash\{h\}$.

We define families $\left\{\mathscr{T}_{\beta}: \beta<2^{\alpha}\right\}$ and filters $\left\{\mathscr{F}_{\beta}: \beta \leqslant 2^{\alpha}\right\}$ such that:

(i) $\mathscr{T}_{\beta} \supset \mathscr{T}_{\xi}$ and $\mathscr{F}_{\beta} \subset \mathscr{F}_{\xi}$ for $\beta<\xi \leqslant 2^{\alpha}$;

(ii) $\left|\mathscr{T} \backslash \mathscr{T}_{\beta}\right| \leqslant \omega+|\beta|$ for $\beta<2^{\alpha}$;

(iii) $\left\langle\varnothing, \mathscr{T}_{\beta}\right\rangle$ is $\left\{b_{\gamma}: \gamma \in \alpha\right\}$-independent $\bmod \mathscr{F}_{\beta}$ for $\beta<2^{\alpha}$;

(iv) $\mathscr{T}_{\beta}=\bigcap_{\xi<\beta} \mathscr{T}_{\xi}$ and $\mathscr{F}_{\beta}=\bigcup_{\xi<\beta} \mathscr{F}_{\xi}$ for each nonzero limit $\beta \leqslant 2^{\alpha}$;

(v) if $d_{\beta} \in B$ for $\beta<2^{\alpha}$, then either $d_{\beta} \in \mathscr{F}_{\beta+1}$ or $1-d_{\beta} \in \mathscr{F}_{\beta+1}$;

(vi) if $d_{\beta}=S$, for $\beta<2^{\alpha}$, where $S$ is a monotone function from $\mathscr{P}_{\omega}(\alpha)$ into $B$ and $S\left[\mathscr{P}_{\omega}(\alpha)\right] \subset \mathscr{F}_{\beta}$, then there is a multiplicative function $T: \mathscr{P}_{\omega}(\alpha) \rightarrow \mathscr{F}_{\beta+1}$ such that $T \leqslant S$.

We proceed by transfinite recursion. For nonzero limit ordinals we define $\mathscr{T}_{\beta}$ and $\mathscr{F}_{\beta}$ by (iv). Now, let $\beta<2^{\alpha}$, and we define $\mathscr{T}_{\beta+1}$ and $\mathscr{F}_{\beta+1}$ according to two cases.

Case 1. Let $d_{\beta} \in B$. If $\mathscr{F}_{\beta} \cup\left\{d_{\beta}\right\}$ generates a filter $\mathscr{F}$, and if $\left\langle\varnothing, \mathscr{T}_{\beta}\right\rangle$ is $\left\{b_{\gamma}\right.$ : $\gamma \in \alpha\}$-independent $\bmod \mathscr{F}$, then let $\mathscr{T}_{\beta+1}=\mathscr{T}_{\beta}$ and let $\mathscr{F}_{\beta+1}=\mathscr{F}$. Otherwise, there is an $n \in \omega$ and sets $\left\{g_{i}: i \in n\right\} \subset \mathscr{T}_{\beta},\left\{\xi_{i}: i \in n\right\} \subset \alpha$, and $b \in \mathscr{F}_{\beta}$ such that $\left\{\gamma: g_{i}(\gamma)=\xi_{i}\right.$ for $i \in n$ and $\left.b \wedge b_{\gamma} \neq 0\right\}=\varnothing$. Observe that $\left\{\gamma: b_{\gamma}<1-b\right\} \supset\{\gamma$ : $g_{i}(\gamma)=\xi_{i}$ for $\left.i \in n\right\}$. It follows that $\left\langle\varnothing, \mathscr{T}_{\beta+1}\right\rangle$ is $\left\{b_{\gamma}: \gamma \in \alpha\right\}$-independent $\bmod \mathscr{F}_{\beta+1}$ where $\mathscr{T}_{\beta+1}=\mathscr{T}_{\beta} \backslash\left\{g_{i}: i \in n\right\}$ and $\mathscr{F}_{\beta+1}$ is the filter generated by $\mathscr{F}_{\beta}$ $\cup\{1-b\}$. Conditions (i) $-($ vi) are easily verified.

Case 2. Let $d_{\beta}=S$, where $S$ is a monotone function from $\mathscr{P}_{\omega}(\alpha)$ into $B$ and $S\left[\mathscr{P}_{\omega}(\alpha)\right] \sqsubset \mathscr{F}_{\beta}$. Choose $g \in \mathscr{F}_{\beta}$ arbitrarily. We will define $T: \mathscr{P}_{\omega}(\alpha) \rightarrow B$ by defining $T(\{\xi\})$ for $\xi<\alpha$ by induction on $\xi$. Fix an indexing $\left\{F_{\delta}: \delta \in \alpha\right\}$ of $\mathscr{P}_{\omega}(\alpha)$. If $F \in \mathscr{P}_{\omega}(\alpha)$ and $T(\{\xi\})$ is defined for $\xi \in F$, then $T(F)=\wedge\{T(\{\xi\}): \xi \in F\}$. As in Kunen's proof, if we could define $T\left(F_{\xi}\right)$ to be the join of $\left\{b_{\gamma} \wedge S\left(F_{\delta}\right): \gamma \in \alpha\right.$, $\delta \in \alpha, F_{\xi} \subseteq F_{\delta}$ and $\left.g(\gamma)=\delta\right\}$, then we would have a multiplicative function refining $S$. However, since this join likely does not exist in $B$, we use the fact that $B$ satisfies $F_{\alpha^{+}}$to patch it up.

Let $T(\varnothing)=1$ and assume that $S(\varnothing)=1$ (this is only for convenience of notation and poses no problem; simply redefine $T(\varnothing)=S(\varnothing)$ at the end). Let $A_{0}=\left\{b_{\gamma} \wedge\right.$ $S\left(F_{g(\gamma)}\right): \gamma \in \alpha$ and $\left.0 \in F_{g(\gamma)}\right\}$ and let $C_{0}=\{1 \backslash S(\{0\})\} \cup\left\{b_{\gamma}: \gamma \in \alpha\right.$ and $0 \notin$ $\left.F_{g(\gamma)}\right\} \cup\left\{b_{\gamma}-S\left(F_{g(\gamma)}\right): \gamma \in \alpha\right.$ and $\left.0 \in F_{g(\gamma)}\right\}$. Since $\left\{b_{\gamma}: \gamma \in \alpha\right\}$ is a cellular 
family and $S$ is monotone, if $a \in A_{0}$ and $c \in C_{0}$, then $a \wedge c=0$. Let $T(\{0\}) \in B$ be such that $a<T(\{0\})$ for $a \in A_{0}$ and $T(\{0\}) \wedge c=0$ for $c \in C_{0}$, which exists because $B$ satisfies $F_{\alpha^{+}}$.

We define recursively, for $\xi<\alpha, T(\{\xi\}) \in B$ so that $a<T(\{\xi\})$ and $c \wedge T(\{\xi\})$ $=0$ for $a \in A_{\xi}$ and $c \in C_{\xi}$, where $A_{\xi}=\left\{b_{\gamma} \wedge S\left(F_{g(\gamma)}\right): \gamma \in \alpha\right.$ and $\left.\xi \in F_{g(\gamma)}\right\}$ and $C_{\xi}=\left\{T(F)-S(F \cup\{\xi\}): \quad F \in \mathscr{P}_{\omega}(\xi)\right\} \cup\left\{b_{\gamma}: \quad \gamma \in \alpha\right.$ and $\left.\xi \notin F_{g(\gamma)}\right\} \cup\left\{b_{\gamma}-\right.$ $S\left(F_{g(\gamma)}\right): \gamma \in \alpha$ and $\left.\xi \in F_{g(\gamma)}\right\}$. Suppose that $\xi<\alpha$ and, for $\beta<\xi, T(\{\beta\})$ has been chosen to satisfy the recursion. Let $A_{\xi}$ and $C_{\xi}$ be as above and let $\gamma_{1} \in \alpha$ be such that $b_{\gamma_{1}} \wedge S\left(F_{g\left(\gamma_{1}\right)}\right)=a \in A_{\xi}$. Let $\gamma_{2}, \gamma_{3} \in \alpha$ and $F \in \mathscr{P}_{\omega}$ ( $\left.\xi\right)$ be such that $\left\{c_{1}, c_{2}, c_{3}\right\} \subset C_{\xi}$ where $c_{1}=T(F)-S(\mathscr{F} \cup\{\xi\}), c_{2}=b_{\gamma_{2}}$ (i.e. $\left.\xi \notin F_{g\left(\gamma_{2}\right)}\right)$ and $c_{3}=b_{\gamma_{3}}-S\left(F_{g\left(\gamma_{3}\right)}\right)$ with $\xi \in F_{g\left(\gamma_{3}\right)}$. In order that $T(\{\xi\})$ can be defined it suffices to show that $a \wedge c_{1}=a \wedge c_{2}=a \wedge c_{3}=0$. Firstly, $a \wedge c_{2}=0$, since $\xi \in$ $F_{g\left(\gamma_{1}\right)} \backslash F_{g\left(\gamma_{2}\right)}$ implies $\gamma_{1} \neq \gamma_{2}$, and $b_{\gamma_{1}} \wedge b_{\gamma_{2}}=0$. Secondly, either $\gamma_{1} \neq \gamma_{3}$ in which case $b_{\gamma_{1}} \wedge b_{\gamma_{3}}=0$ or $\gamma_{1}=\gamma_{3}$ and $a<S\left(F_{g\left(\gamma_{1}\right)}\right)$ and $c_{3} \wedge S\left(F_{g\left(\gamma_{1}\right)}\right)=0$, from which it follows that $a \wedge c_{3}=0$. Finally, we show that $a \wedge c_{1}=0$. First observe that, by the recursion, if $b_{\gamma_{1}} \wedge T(F) \neq 0$, then $F \subset F_{g\left(\gamma_{1}\right)}$ (i.e. it must be the case that $b_{\gamma_{1}} \wedge S\left(F_{g\left(\gamma_{1}\right)}\right) \in A_{\delta}$ for $\left.\delta \in F\right)$. Now, since $F \subset F_{g\left(\gamma_{1}\right)}$ and $\xi \in F_{g\left(\gamma_{1}\right)}, F \cup\{\xi\} \subset$ $F_{g\left(\gamma_{1}\right)}$, and so $S\left(F_{g\left(\gamma_{1}\right)}\right)<S(F \cup\{\xi\})$ since $S$ is monotone. Therefore, $a \wedge c_{1}=b_{\gamma_{1}}$ $\wedge S\left(F_{g\left(\gamma_{1}\right)}\right) \wedge T(F)-S(F \cup\{\xi\}) \leqslant S\left(F_{g\left(\gamma_{1}\right)}\right)-S(F \cup\{\xi\})=0$. To show that $T$ refines $S$ it suffices to show that, for any $\xi \in \alpha$ and $F \in \mathscr{P}_{\omega}(\xi), T(F) \leqslant S(F)$ implies $T(F \cup\{\xi\}) \leqslant S(F \cup\{\xi\})$. But, of course, $T(F)-S(F \cup\{\xi\}) \in C_{\xi}$ implies that $T(\{\xi\}) \wedge T(F) \leqslant S(F \cup\{\xi\})$.

To complete Case 2, we let $\mathscr{T}_{\beta+1}=\mathscr{T}_{\beta} \backslash\{g\}$ and let $\mathscr{F}_{\beta+1}$ be the filter generated by $\mathscr{F}_{\beta} \cup T\left[\mathscr{P}_{\omega}(\alpha)\right]$. The fact that $\mathscr{F}_{\beta+1}$ is a filter will be a consequence of the only thing that remains to be shown: $\left\langle\varnothing, \mathscr{T}_{\beta+1}\right\rangle$ is $\left\{b_{\gamma}: \gamma \in \alpha\right\}$-independent with respect to $\mathscr{F}_{\beta+1}$. Let $G \in \mathscr{P}_{\omega}\left(\mathscr{T}_{\beta+1}\right), t \in \alpha^{G}, F_{\delta} \in \mathscr{P}_{\omega}(\alpha)$ and $b \in \mathscr{F}_{\beta}$; we must show that $\left\{\gamma \in \alpha: g^{\prime}(\gamma)=t\left(g^{\prime}\right)\right.$ for $g^{\prime} \in G$ and $\left.b \wedge T\left(F_{\delta}\right) \wedge b_{\gamma} \neq 0\right\} \neq \varnothing$. For each $\gamma \in \alpha$ such that $g(\gamma)=\delta$ and $\xi \in F_{\delta}, T(\{\xi\})>b_{\gamma} \wedge S\left(F_{g(\gamma)}\right)$ since $b_{\gamma} \wedge S\left(F_{g(\gamma)}\right) \in A_{\xi}$. Therefore $\left\{\gamma \in \alpha: g^{\prime}(\gamma)=t\left(g^{\prime}\right)\right.$ for $g^{\prime} \in G$ and $\left.b \wedge T\left(F_{\delta}\right) \wedge b_{\gamma} \neq 0\right\} \supset\{\gamma \in \alpha$ : $g^{\prime}(\gamma)=t\left(g^{\prime}\right)$ for $g^{\prime} \in G, g(\gamma)=\delta$ and $\left.b \wedge S\left(F_{\delta}\right) \wedge b_{\gamma} \neq 0\right\} \neq \varnothing$ since $b \wedge S\left(F_{\delta}\right)$ $\in \mathscr{F}_{\beta}$.

This completes the recursion and the proof of the theorem since $\mathscr{F}=\mathscr{F}_{2^{\alpha}}$ is an ultrafilter by (v), $\alpha^{+}$-good by (vi), and $(\eta, \omega)$-uniform since $\mathscr{F} \supset \mathscr{F}_{0}$.

3.3. Remark. The construction of an $(\eta, \omega)$-uniform $\alpha^{+}$-good ultrafilter on a boolean algebra of cardinality $2^{\alpha}$ which satisfies $F_{\alpha^{+}}$and $E_{\alpha}$ is an improvement of some of the results of Negrepontis in [N3]. In the first place we do not need any special assumptions on the cardinal $\alpha$ whereas for Negrepontis' results to apply we would have to assume $2^{\alpha}=\alpha^{+}$. In addition, we do not require that the boolean algebra satisfies condition $H_{\alpha^{+}}$. However, Negrepontis also constructs $\alpha$-good ultrafilters of boolean algebras of cardinality $\alpha$ which satisfy $H_{\alpha}$ where $\alpha$ satisfies $\alpha=\alpha^{\alpha}$.

3.4. TheOREM. For any cardinal $\eta \leqslant \alpha$, there is an $(\eta, \omega)$-uniform $\alpha^{+}-O K$ ultrafilter on $B$.

Proof. Let $\mathscr{D}$ be the set which is the union of $B$ with $\{S: S$ is a monotone function from $\omega$ into $B\}$. Fix an indexing $\left\{d_{\xi}: \xi<2^{\alpha}\right\}$ so that each monotone 
function $S: \omega \rightarrow B$ appears $2^{\alpha}$ times. Just as in Theorem 3.2 choose $h \in \mathscr{T}$, define $\left\{c_{\gamma}: \gamma<\eta\right\} \subset B$ and $\left\{c_{\gamma, m}: \gamma<\eta, m \in \omega\right\} \subset B$. Let $\mathscr{F}_{0}$ be the filter generated by $\left\{c_{\gamma, m}: \gamma<\eta, m \in \omega\right\} \cup\left\{b \in B: b_{\gamma}<b\right.$ for all $\left.\gamma<\alpha\right\}$. It is easily checked that $\left\langle\left\{A_{f, \beta}: f \in \mathscr{S}, \beta \in \alpha^{+}\right\}, \mathscr{T} \backslash\{h\}\right\rangle$ is $\left\{b_{\gamma}: \gamma \in \alpha\right\}$-independent $\bmod \mathscr{F}_{0}$. Let $\mathscr{S}_{0}=$ $\mathscr{S}$.

We define families $\left\{\mathscr{S}_{\beta}: \beta \leqslant 2^{\alpha}\right\}$ and filters $\left\{\mathscr{F}_{\beta}: \beta \leqslant 2^{\alpha}\right\}$ such that:

(i) $\mathscr{S}_{\beta} \supset \mathscr{S}_{\xi}$ and $\mathscr{F}_{\beta} \subset \mathscr{F}_{\xi}$ for $\beta<\xi \leqslant 2^{\alpha}$;

(ii) $\left|\mathscr{S} \backslash \mathscr{S}_{\beta}\right| \leqslant \omega+|\beta|$ for $\beta<2^{\alpha}$;

(iii) $\left\langle\left\{A_{f, \delta}: f \in \mathscr{S}_{\beta}, \delta \in \alpha^{+}\right\}, \varnothing\right\rangle$ is $\left\{b_{\gamma}: \gamma \in \alpha\right\}$-independent $\bmod \mathscr{F}_{\beta}$ for $\beta<2^{\alpha}$;

(iv) $\mathscr{F}_{\beta}=\bigcup_{\xi<\beta} \mathscr{F}_{\xi}$ and $\mathscr{S}_{\beta}=\bigcap_{\zeta<\beta} \mathscr{S}_{\zeta}$ for each nonzero limit ordinal $\beta \leqslant 2^{\alpha}$;

(v) if $d_{\beta} \in B$ for $\beta<2^{\alpha}$, then either $d_{\beta} \in \mathscr{F}_{\beta+1}$ or $1-d_{\beta} \in \mathscr{F}_{\beta+1}$;

(vi) if $d_{\beta}=S$ for $\beta<2^{\alpha}$, where $S$ is a monotone function from $\omega$ into $B$ and $S[\omega] \subset \mathscr{F}_{\beta}$, then there is a monotone function $T$ from $\mathscr{P}_{\omega}\left(\alpha^{+}\right)$into $\mathscr{F}_{\beta+1}$ such that, for $F \in \mathscr{P}_{\omega}\left(\alpha^{+}\right)-\{\varnothing\}$,

$$
T(F)=\bigwedge\{T\{\xi\}: \xi \in F\} \leqslant S(|F|-1) .
$$

We proceed by transfinite recursion. For nonzero limit ordinals, $\beta \leqslant 2^{\alpha}$, we define $\mathscr{S}_{\beta}$ and $\mathscr{F}_{\beta}$ by (iv). Now, let $\beta<2^{\alpha}$, and we define $\mathscr{I}_{\beta+1}$ and $\mathscr{F}_{\beta+1}$ according to two cases.

Case 1. Let $d_{\beta} \in B$. If $\mathscr{F}_{\beta} \cup\left\{d_{\beta}\right\}$ generates a filter $\mathscr{F}$, and if $\left\langle\left\{A_{f, \delta}: f \in \mathscr{S}_{\beta}\right.\right.$, $\left.\delta\left\langle\alpha^{+}\right\}, \varnothing\right\rangle$ is $\left\{b_{\gamma}: \gamma \in \alpha\right\}$-independent $\bmod \mathscr{F}$, then let $\mathscr{S}_{\beta+1}=\mathscr{S}_{\beta}$ and $\mathscr{F}_{\beta+1}=$ $\mathscr{F}$. Otherwise, there is an $n \in \omega$ and sets $\left\{f_{i}: i \in n\right\} \subset \mathscr{P}_{\beta},\left\{\xi_{i}: i \in n\right\} \subset \alpha^{+}$and $b \in \mathscr{F}_{\beta}$ such that

$$
\left\{\gamma: b \wedge b_{\gamma} \wedge \bigwedge_{i \in n} A_{f, \xi_{i}} \neq 0\right\}=\varnothing
$$

It follows that $\left\langle\left\{A_{f, \delta}: f \in \mathscr{S}_{\beta+1}, \delta \in \alpha^{+}\right\}, \quad \varnothing\right\rangle$ is $\left\{b_{\gamma}: \quad \gamma \in \alpha\right\}$-independent $\bmod \mathscr{F}_{\beta+1}$, where $\mathscr{S}_{\beta+1}=\mathscr{S}_{\beta}-\left\{f_{i}: i \in n\right\}$ and $\mathscr{F}_{\beta+1}$ is the filter generated by $\mathscr{F}_{\beta}$ $\cup\{1-b\}$.

Case 2. Let $d_{\beta}=S$ where $S$ is a monotone function from $\omega$ into $B$ and $S[\omega] \subset$ $\mathscr{F}_{\beta}$. Choose a countably infinite subset $\left\{f_{i}: i \in \omega\right\}$ of $\mathscr{S}_{\beta}$ and let it be faithfully indexed. We will define $T: \alpha^{+} \rightarrow B$ by recursion. Since $\mathscr{F}_{0} \subset \mathscr{F}_{\beta}$ we may assume that $S(n) \leqslant c_{0, n}$ for $0<n \in \omega$ because if $T$ satisfies (vi) for $S^{\prime}$, where $S^{\prime}(n)=S(n)$ $\wedge c_{0, n}$, then it satisfies (vi) for $S$. Again the idea is to mimick Kunen's construction of $c$-OK points. We would like to define $T(\{\delta\})$ to be the join of

$$
\left\{S(k)-S(k+1) \wedge \bigvee\left\{A_{f_{i}, \delta}: i \leqslant k\right\}: k \in \omega\right\}
$$

which we can only do if $B$ is $\sigma$-complete. However, we again use the fact that $B$ satisfies $F_{\alpha^{+}}$and construct $T$ by recursion. As in 3.2 we assume that if $F \in \mathscr{P}_{\omega}\left(\alpha^{+}\right)$ and $T(\{\xi\})$ is defined for $\xi \in F$ then

$$
T(F)=\bigwedge\{T(\{\xi\}): \xi \in F\} .
$$

Let $T(\varnothing)=1$ (again only a notational convenience as in 3.2). Let

$$
A_{0}=\left\{S(k)-S(k+1) \wedge \bigvee\left\{A_{f_{i}, 0}: i \leqslant k\right\}: k \in \omega\right\}
$$


and let

$$
C_{0}=\left\{(S(k)-S(k+1))-\bigvee\left\{A_{f_{i}, 0}: i \leqslant k: k \in \omega\right\} \cup\{1-S(0)\} .\right.
$$

Choose $T(\{0\}) \in B$ such that $a \leqslant T(\{0\})$ for $a \in A_{0}$ and $c \wedge T(\{0\})=0$ for $c \in C_{0}$.

For $\delta<\alpha^{+}$, we define recursively $T(\{\delta\}) \in B$ so that $a<T(\{\delta\})$ and $c \wedge$ $T(\{\delta\})=0$ for $a \in A_{\delta}$ and $c \in C_{\delta}$, where

$$
A_{\delta}=\left\{S(k)-S(k+1) \wedge \bigvee\left\{A_{f_{i}, \delta}: i \leqslant k\right\}: k \in \omega\right\}
$$

and

$$
\begin{aligned}
C_{\delta}= & \left\{(S(k)-S(k+1))-\bigvee\left\{A_{f_{1}, \delta}: i \leqslant k\right\}: k \in \omega\right\} \\
& \cup\left\{T(F)-S(|F \cup\{\delta\}|-1): F \in \mathscr{P}_{\omega}(\delta)\right\} .
\end{aligned}
$$

Suppose that $\delta<\alpha$ and, for $\xi<\delta, T(\{\xi\})$ has been chosen to satisfy the recursion. Let $A_{\delta}$ and $C_{\delta}$ be as above and let $a \in A_{\delta}$ and $c \in C_{\delta}$. We would like to show that $a \wedge c=0$. Since $S$ is monotone the only nontrivial case is

$$
a=S(k)-S(k+1) \wedge \bigvee\left\{A_{f_{1}, \delta}: i \leqslant k\right\}
$$

and $c=T(F)-S(|F \cup\{\delta\}|-1)$ for some $F \in \mathscr{P}_{\omega}(\delta)$ with $k<|F|$. By the definition of $T(F)$,

$$
\begin{aligned}
a \wedge T(F) \wedge S(k)-S(k+1) \leqslant & \bigwedge\left\{\bigvee\left\{A_{f_{1}, \xi}: i \leqslant k\right\}: \xi \in F \cup\{\delta\}\right\} \\
& \wedge S(k)-S(k+1)
\end{aligned}
$$

However, $\wedge\left\{\bigvee\left\{A_{f_{i}, \xi}: i \leqslant k\right\}: \xi \in F \cup\{\delta\}\right\}=\bigvee\left\{\wedge\left\{A_{f_{r(\xi)}, \xi}: \xi \in F \cup\{\delta\}\right\}: r\right.$ is a function from $F \cup\{\delta\}$ into $\{0,1, \ldots k\}\}$. However, for each $r \in(k+1)^{F \cup\{\delta\}}$,

$$
\bigwedge\left\{A_{f_{r(\xi)}, \xi}: \xi \in F \cup\{\xi\}\right\}=0 .
$$

Indeed, $|F \cup\{\delta\}|>k+1$ and so, for some $\xi \neq \xi^{\prime}$ with $\left\{\xi, \xi^{\prime}\right\} \subset F \cup\{\delta\}$, we have $r(\xi)=r\left(\xi^{\prime}\right)$, but $A_{f_{r(\xi), \xi}} \wedge A_{f_{r\left(\xi^{\prime}\right)}, \xi^{\prime}}=0$ by definition of an independent matrix. Now, let $\mathscr{S}_{\beta+1}=\mathscr{S}_{\beta}-\left\{f_{i}: i \in \omega\right\}$ and let $\mathscr{F}_{\beta+1}$ be the filter generated by $\mathscr{F}_{\beta} \cup T\left[\mathscr{P}_{\omega}\left(\alpha^{+}\right)\right]$. It remains only to show that $\left\langle\left\{A_{f, \delta}: f \in \mathscr{S}_{\beta+1}, \delta \in \alpha^{+}\right\}, \varnothing\right\rangle$ is $\left\{b_{\gamma}: \gamma \in \alpha\right\}$-independent with respect to $\mathscr{F}_{\beta+1}$ (again, that $\mathscr{F}_{\beta+1}$ is a filter also follows from this). To show this, let $b \in \mathscr{F}_{\beta}$ and $\xi_{0}<\xi_{1}<\cdots<\xi_{n-1}<\alpha^{+}$be arbitrary. Note that for $\gamma<\alpha$

$$
b_{\gamma} \wedge T\left(\left\{\xi_{0}, \ldots, \xi_{n-1}\right\}\right) \geqslant b_{\gamma} \wedge S(n) \wedge \wedge\left\{A_{f_{i}, \xi_{i}}: i<n\right\}
$$

since $b_{\gamma} \wedge S(k)=0$ for some $k \in \omega$. Therefore, for any finite $F \subset \mathscr{S}_{\beta+1}$ and $t \in\left(\alpha^{+}\right)^{F}$

$$
\begin{aligned}
& \left\{\gamma \in \alpha: b_{\gamma} \wedge T\left(\left\{\xi_{0}, \ldots, \xi_{n-1}\right\}\right) \wedge b \wedge \wedge\left\{A_{f, t(f)}: f \in F\right\} \neq 0\right\} \\
& \quad \supset\left\{\gamma \in \alpha: b_{\gamma} \wedge b \wedge s(n) \wedge \wedge\left\{A_{f_{i}, \xi_{i}}: i<n\right\} \wedge \wedge\left\{A_{f, t(f)}: f \in F\right\} \neq 0\right\} \neq \varnothing .
\end{aligned}
$$

The recursion is complete and we let $\mathscr{F}=\mathscr{F}_{2^{\alpha}}$. It follows that $\mathscr{F}$ is as desired since condition (vi) is obviously just a translation of the definition of $\alpha^{+}-\mathrm{OK}$.

3.5. THEOREM. For any infinite cardinal $\eta \leqslant \alpha$, there is an $(\eta, \omega)$-uniform, $\alpha^{+}$-good, $\alpha^{+}-$OK ultrafilter $\mathscr{F}$ of $B$. 
Proof. We just do the recursions of Theorems 3.2 and 3.4 simultaneously, that is, alternatingly.

In his paper [Ke1], Keisler defines the degree of goodness of an ultrafilter $\mathscr{F}$ by $G(\mathscr{F})=\min \left\{\alpha: \mathscr{F}\right.$ is not $\alpha^{+}$-good $\}$. There is an interesting way to construct an ultrafilter $\mathscr{F}$ on $B$ so that $G(\mathscr{F})=\beta^{+}$for any fixed $\beta \leqslant \alpha$. This is an easy generalization of a result of Keisler's (see [Ke2 or CN]).

3.6. ThEOREM. For any $\beta \leqslant \alpha$, there is an ultrafilter $\mathscr{F}$ of $B$ such that $G(\mathscr{F})=\beta^{+}$.

Proof. Recall that $\left\{b_{\gamma}: \gamma<\beta\right\}$ is a cellular family of $B$. Fix ultrafilters $\left\{\mathscr{F}_{\gamma}\right.$ : $\gamma<\beta\}$ such that $b_{\gamma} \in \mathscr{F}_{\gamma}$ for $\gamma<\beta$. Let $P$ be a countably incomplete $\beta^{+}$-good ultrafilter on $\beta$. Define $\mathscr{F}=\left\{b \in B:\left\{\gamma<\beta: b \wedge b_{\gamma} \in \mathscr{F}_{\gamma}\right\} \in P\right\}$; we shall show that $\mathscr{F}$ is $\beta^{+}$-good. We first observe that if $a_{\gamma} \in \mathscr{F}_{\gamma}$ for $\gamma \in A$ with $A \in P$, then there is a $b \in B$ satisfying simultaneously $A=\left\{\gamma<\beta: b \wedge b_{\gamma}=a_{\gamma} \wedge b_{\gamma}\right\}$ and $\beta \backslash A=\left\{\gamma<\beta: b \wedge b_{\gamma}=0\right\}$ since $B$ satisfies $F_{\alpha^{+}}$. Let $S: \mathscr{P}_{\omega}(\beta) \rightarrow \mathscr{F}$ be a monotone function and let $\left\{A_{n}: n \in \omega\right\} \subset P$ be such that $\cap A_{n}=\varnothing$. Define $S^{\prime}=\mathscr{P}_{\omega}(\beta) \rightarrow P$ by $S^{\prime}(F)=\left\{\gamma \in \beta: S(F) \in \mathscr{F}_{\gamma}\right\} \cap A_{|F|}$ for $F \in \mathscr{P}_{\omega}(\beta)$. Let $T^{\prime}$ : $\mathscr{P}_{\omega}(\beta) \rightarrow P$ be multiplicative and $T^{\prime} \leqslant S^{\prime}$. For each $\gamma \in \beta$, let $F_{\gamma}=\{\delta \in \beta$ : $\left.\gamma \in T^{\prime}(\{\delta\})\right\} ;\left|F_{\gamma}\right|<\omega$ since $\cap A_{n}=\varnothing$. Define, for $\gamma \in \beta, a_{\gamma}=S\left(F_{\gamma}\right) \wedge b_{\gamma}$. Define $T: \mathscr{P}_{\omega}(\beta) \rightarrow \mathscr{F}$ by the following recursion. For each $\gamma<\beta, b_{\xi} \wedge T(\{\gamma\})=a_{\xi}$ for $\xi \in T^{\prime}(\{\gamma\})$, and $c \wedge T(\{\gamma\})=0$ for $c \in\left\{b_{\xi}: \xi \in \beta \backslash T^{\prime}(\{\xi\})\right\} \cup\{T(F)-$ $\left.S(F \cup\{\gamma\}): F \in \mathscr{P}_{\omega}(\gamma)\right\}$. That this recursion may be carried out follows from the fact that if $\xi \in T^{\prime}(\{\gamma\}), F \in \mathscr{P}_{\omega}(\gamma)$ and $b_{\xi} \wedge T(F) \neq 0$, then $F \cup\{\gamma\} \subset F_{\xi}$ and $a_{\xi} \leqslant S(F \cup\{\gamma\})$. As in Theorem 3.2, $T$ is multiplicative and so $\mathscr{F}$ is $\beta^{+}$-good. It remains to show that $\mathscr{F}$ is not $\beta^{++}$-good. Choose $\left\{a_{n}: n \in \omega\right\} \subset \mathscr{F}$ such that $A_{n} \supset\left\{\gamma \in \beta: b_{\gamma} \wedge a_{n} \neq 0\right\}$. Define $S: \mathscr{P}_{\omega}\left(\beta^{+}\right) \rightarrow \mathscr{F}$ by $S(F)=a_{|F|}$. Suppose $T$ : $\mathscr{P}_{\omega}\left(\beta^{+}\right) \rightarrow \mathscr{F}$ is multiplicative. Let $T^{\prime}(\xi)=\left\{\gamma \in \beta: T(\xi) \in \mathscr{F}_{\gamma}\right\}$ for $\xi<\beta^{+}$; by the definition of $\mathscr{F}, T^{\prime}(\xi) \in P$ for each $\xi<\beta^{+}$. Clearly, for some $\gamma \in \beta$, there is an infinite set $\left\{\xi_{i}: i \in \omega\right\} \subset \beta^{+}$such that $\gamma \in \bigcap\left\{T^{\prime}\left(\xi_{i}\right): i \in \omega\right\}$. However, for some $n \in \omega, \gamma \notin A_{n}$ and $b_{\gamma} \wedge a_{n}=0$. Therefore, $T\left(\left\{\xi_{i}: i \in n\right\}\right) \nless S\left(\left\{\xi_{i}: i \in n\right\}\right)$ since

$$
T\left(\left\{\xi_{i}: i \in n\right\}\right)=\bigwedge\left\{T\left(\xi_{i}\right): i \in n\right\} \in \mathscr{F}_{\gamma} .
$$

We shall now show, with a cardinal assumption, that for any boolean algebra $\mathscr{C}$ and any $(\omega, \omega)$-uniform ultrafilter $\mathscr{F}$ on $\mathscr{C}$ that $G(\mathscr{F}) \leqslant|\mathscr{C}|$. This answers a question in $[\mathbf{N}]$ and in [Ke1].

3.7. THEOREM. Let $\eta$ be a cardinal satisfying $\eta^{\omega}=\eta$ and let $\mathscr{C}$ be a boolean algebra of cardinality $\eta$. If $\mathscr{F}$ is an $(\omega, \omega)$-uniform ultrafilter on $\mathscr{C}$ then $G(\mathscr{F}) \leqslant \eta$.

Proof. Let $\left\{g_{\xi}: \omega<\xi<\eta\right\}$ be a listing of all monotone functions from $\mathscr{P}_{\omega}(\omega)$ into $\mathscr{F}$. Since $\mathscr{F}$ is $(\omega, \omega)$-uniform there is a cellular family $\left\{c_{n}: n \in \omega\right\} \subset \mathscr{C}$ such that, for each $b \in \mathscr{F},\left|\left\{n \in \omega: b \wedge c_{n} \neq 0\right\}\right|=\omega$. We define a function $f$ : $\mathscr{P}_{\omega}(\eta) \rightarrow \mathscr{F}$ as follows. For $F \in \mathscr{P}_{\omega}(\omega)$, let

$$
f(F)=1-\bigvee\left\{c_{n}: n \leqslant \max (F)\right\} \in \mathscr{F} \text {. }
$$

For $F \in \mathscr{P}_{\omega}(\omega)$ and $\omega \leqslant \xi<\eta$, let

$$
f(F \cup\{\xi\})=g_{\xi}(F \cup\{1+\max (F)\}) .
$$


Finally for $F \in \mathscr{P}_{\omega}(\eta)$, let

$$
f(F)=\bigwedge\{f(F \cap(\omega \cup\{\xi\})): \omega \leqslant \xi<\eta \text { and } \xi \in F\} .
$$

It is easily checked that $f$ is a well-defined monotone function from $\mathscr{P}_{\omega}(\eta)$ into $\mathscr{F}$. Suppose $g: \mathscr{P}_{\omega}(\eta) \rightarrow \mathscr{F}$ is a multiplicative function which refines $f$. Let $\omega \leqslant \xi<\eta$ be such that $g_{\xi}=\left.g\right|_{\mathscr{P}_{\omega}(\omega)}$. For each $n \in \omega, f(n \cup\{\xi\})=g_{\xi}(n+1)=g(n+1) \geqslant$ $g(n \cup\{\xi\})=g(n) \wedge g(\{\xi\})$. Therefore, since $g(n) \geqslant g(n+1), g(\{\xi\}) \leqslant g(n)$ for $n \in \omega$. However, as $g$ refines $f, g(n) \wedge c_{k}=0$ for $k<n$ which implies that $g(\{\xi\}) \wedge c_{k}=0$ for $k \in \omega$. This contradicts that $g(\{\xi\}) \in \mathscr{F}$.

We do not know if the assumption that $\eta^{\omega}=\eta$ is necessary in 3.7 but $(\omega, \omega)$-uniformity is necessary, for if $\mathscr{F}$ is a $P_{\alpha}$-point, then it is $\alpha^{+}$-good. In any case, we see in 4.8 that no ultrafilter is $\eta^{++}$-good.

3.8. Corollary. If $2^{\omega}=\omega_{1}$, then there are no $(\omega, \omega)$-uniform $\omega_{2}$-good ultrafilters on $\mathscr{P}(\omega) / \mathscr{P}_{\omega}(\omega)$.

We state Corollary 3.8 explicitly because we show in 3.11 that the assumption $2^{\omega}=\omega_{1}$ is necessary. First we remind the reader of the definition of Martin's Axiom for $\sigma$-centred posets of size $\omega_{1}, \mathrm{MA}_{\omega_{1}}(\Sigma)$. A poset $(P, \leqslant)$ is $\sigma$-centred if $P=$ $\cup\left\{P_{n}: n \in \omega\right\}$, where for any $n \in \omega$ and finite $F \subset P_{n}$ there is an $r \in P$ with $r \leqslant p$ for $p \in F$. A set $D \subset P$ is called dense if for any $p \in P$ there is a $d \in D$ with $d \leqslant p$. A set $G \subset P$ is called directed if for $g_{1}, g_{2} \in G$ there is $g \in G$ with $g \leqslant g_{1}$ and $g \leqslant g_{2}$. MA( $\sigma$-centred) is the statement that for any $\sigma$-centred poset $(P,<)$ and fewer than $c$ dense subsets $\mathscr{C}$ there is a directed $G \subset P$ with the property that for each $D \in \mathscr{C}$ there are $d \in D$ and $g \in G$ with $g \leqslant d$.

3.9. THEOREM. MA( $\sigma$-centred $)$ implies there is a c-good ultrafilter on $\mathscr{P}(\omega) / \mathscr{P}_{\omega}(\omega)$ which is not a P-point.

Proof. We fix a partition of $\omega$ into infinitely many disjoint infinite sets $\underline{A}$. We define an ultrafilter $\underline{F}$ in $c$-stages so that for each $F \in \underline{F}$ there is an $A \in \underline{A}$ with $A \cap F$ infinite and so that $\omega \backslash A \in \underline{F}$ for each $A \in \underline{A}$. At each stage of the induction we are considering a filter base of size less than $c$ and a function $g$ from $\mathscr{P}_{\omega}(\alpha)$ into this filter base where $\alpha$ is less than $c$. We "force" an $f$ to refine $g$ in a natural way. Let $P$ be the set of all finite functions from a subset of $\alpha$ to the set of finite subsets of $\omega$. We define $p<q$ provided $p(i) \supset q(i)$ for each $i$ in the domain of $q$ and, for each $S \subset \operatorname{dom}(q), \bigcap\{p(i): i \in S\} \backslash g(S) \subset n$, where $q(i) \subset n$ for all $i$. If $P(n)=\{p: n=\min \{k: p(i) \subset k$ for all $i\}\}$ then $P(n)$ is centred and $P=\cup_{n} P(n)$.

For each $S \in \mathscr{P}_{\omega}(\alpha), F \in \underline{F}$ and $A \in \underline{A}$ such that $g(S) \cap F \cap A$ is infinite, let $D(F, S, A)$ be the dense set of all $p$ such that $F \cap A \cap \cap\{(i): i \in S\}$ is not empty. If $G$ is the set given by MA, then $f(i)=\bigcup\{p(i): p \in G\}$ defines the desired refinement of $g$.

3.10. REMARK. In 3.5 we constructed ultrafilters which are $\alpha^{+}$-good and $\alpha^{+}$-OK. We shall see in 4.4 that the filters constructed in 3.6 are not $\beta^{+}-\mathrm{OK}$; hence, we can construct ultrafilters which are $\alpha^{+}$-good and not $\alpha^{+}-\mathrm{OK}$. However, we have not been able to construct ultrafilters which are $\alpha^{+}$-OK and not $\alpha^{+}$-good. It is possible that a 
technique such as that in 3.7 will accomplish this. That is, define a suitable monotone function $f: \mathscr{P}_{\omega}(\alpha) \rightarrow B$ and carry through the construction of 3.4 . However one must be careful to preserve the independence of the matrix mod $\mathscr{F}_{\xi}$. Another possibility is that 4.6 may be useful.

4. The topological properties. In this section we will investigate some of the topological properties of the Stone space of a boolean algebra using the results of $\S 3$. First we briefly remind the reader of the duality between boolean algebras and compact topological spaces for which the open and closed sets form a base for the topology. (We call such a space zero-dimensional.) As mentioned in the introduction, for a boolean algebra we have an associated topology on the set of ultrafilters- the Stone space. Conversely, let $X$ be a compact zero-dimensional space and let $B$ be the boolean algebra of clopen subsets of $X$ with the operations of intersection and union. Then $X$ is homeomorphic to the Stone space of $B$. Throughout this section we assume that $B$ is a boolean algebra, $X=S(B)$ and all hypothesized sets have cardinality less than the first measurable cardinal. The reason for this last assumption is to ensure that ultrafilters will be countably incomplete. Recall that we defined, for $b \in B$, the clopen subset $\bar{b}=\{\mathscr{F} \in S(B): b \in \mathscr{F}\}$.

A zero-dimensional space $E$ is called extremally disconnected if the clopen subsets of $E$ form a complete boolean algebra (this is equivalent to the property that the closure of each open set is open). Because of our assumption on cardinals, each free ultrafilter of a complete boolean algebra is $(\omega, \omega)$-uniform [GJ, 12H]. A set $D \subset S(B)$ is called strongly discrete is there is a cellular family $\left\{b_{d}: d \in D\right\} \subset B$ with $b_{d} \in d$ for $d \in D$. A subspace $E \subset X$ is said to be $C^{*}$-embedded in $X$ if for each bounded real-valued continuous function $f$ on $E$ there is a continuous real-valued function $g$ on $X$ satisfying $g(x)=f(x)$ for each $x \in E$. The cellularity of a space $Y, c(Y)$ is the supremum of all cardinalities of families of pairwise disjoint nonempty open subsets of $Y$.

The proof of the following lemma is straightforward and is omitted.

4.1. Lemma. An extremally disconnected subspace $E$ of $X$ is $C^{*}$-embedded in $X$ if and only if for each $p \in \mathrm{cl}_{X} E,\{\bar{b} \cap E: b \in p\}$ is an ultrafilter on the boolean algebra of clopen subsets of $E$ (henceforth $\mathrm{CO}(E)$ ).

4.2. TheOrem. Let $\alpha$ be an infinite cardinal and let $p \in X$ be $\alpha^{+}-O K$ (on $B$ ). Then $p \notin \mathrm{cl}_{X} E$ for any $E \subset X \backslash\{p\}$ such that $E$ is $C^{*}$-embedded, extremally disconnected and $c(E) \leqslant \alpha$.

Proof. Let $E$ be a subspace of $X \backslash\{p\}$ satisfying the above conditions. Suppose that $p \in \operatorname{cl}_{X} E$ and let $\mathscr{F}=\{\bar{b} \cap E: b \in p\}$. Since $\mathscr{F}$ is an ultrafilter on the complete algebra $\mathrm{CO}(E), \mathscr{F}$ is $(\omega, \omega)$-uniform. It follows that we may choose $\left\{b_{n}\right.$ : $n \in \omega\} \subseteq p$ so that, for each $b \in p,\left\{n: \bar{b} \cap \bar{b}_{n} \backslash \bar{b}_{n+1} \cap E \neq \varnothing\right\}$ is infinite. Also, since $p$ is $\alpha^{+}-\mathrm{OK}$, there is a function $f: \alpha^{+} \rightarrow p$ such that for each $F \in \mathscr{P}_{\omega}\left(\alpha^{+}\right)$

$$
\bigwedge\{f(\gamma): \gamma \in F\} \leqslant b_{|F|} \text {. }
$$

We note that if we choose $F, G \in \mathscr{P}_{\omega}\left(\alpha^{+}\right)$with $0=|F \cap G|<|F|=|G|=n$ then

$$
\left.\bigwedge\{f(\gamma): \gamma \in F\}-b_{n+1}\right) \wedge\left(\bigwedge\{f(\gamma): \gamma \in G\}-b_{n+1}\right)=0 \text {. }
$$


From this and the fact that $c(E)<\alpha^{+}$, it follows that, for each $n \in \omega$, there is a $\lambda_{n}<\alpha^{+}$such that, for $F \subset \alpha^{+} \backslash \lambda_{n}$ with $|F|=n$,

$$
\overline{\left(\bigwedge\{f(\gamma): \gamma \in F\}-b_{n+1}\right)} \cap E=\varnothing .
$$

Now, from the fact that for $\gamma \in \alpha^{+}$there is an $n \in \omega$ so that $\overline{f(\gamma)-b_{n}} \cap E \neq \varnothing$, it follows that we can find an $n \in \omega$ and finite sets $\left\{F_{\delta}: \delta \in \alpha^{+}\right\}$satisfying

$$
\frac{\overline{\bigwedge\left\{f(\gamma): \gamma \in F_{\delta}\right\}-b_{n}} \cap E \neq \varnothing,}{\bigwedge\left\{f(\gamma): \gamma \in F_{\delta}\right\}-b_{n}} \cap \overline{\bigwedge\left\{f(\gamma): \gamma \in F_{\delta^{\prime}}\right\}} \cap E=\varnothing,
$$

for $\delta<\delta^{\prime}<\alpha^{+}$. However, this contradicts the assumption that $c(E)<\alpha^{+}$. Therefore $p \notin \mathrm{cl}_{X} E$.

The following corollary is probably more appealing for the case when $B=$ $\mathscr{P}(\alpha) / \mathscr{P}_{\alpha}(\alpha)$.

4.3. Corollary. If $B$ satisfies condition $F_{\alpha^{+}}$(we call $X$ an $F_{\alpha^{+}}$-space) and $p \in X$ is an $\alpha^{+}$-OK point then $p$ is not the limit point of any strongly discrete subset $D \subset X$ with $|D|<\alpha^{+}$.

Proof. Let $D \subset X$ be strongly discrete and $|D|<\alpha^{+}$. Since $\operatorname{CO}(D)=\mathscr{P}(D), D$ is extremally disconnected. Since $B$ satisfies condition $F_{\alpha^{+}}$and $D$ is strongly discrete, $D$ is $C^{*}$-embedded in $X$ by 4.1. Therefore $p \notin \mathrm{cl}_{X} D$ by 4.2.

A more general result can be proved using exactly the same proof as that of 4.2.

4.4. Corollary. Let $B$ satisfy condition $F_{\alpha^{+}}$and let $p \in X$ be an $\alpha^{+}-O K$ point. If $\left\{b_{\gamma}: \gamma \in \alpha\right\} \subset B \backslash p$ is a cellular family, then for any $D \subset \cup\left\{\bar{b}_{\gamma}: \gamma \in \alpha\right\}$ with $|D|<\alpha^{+}, p \notin \mathrm{cl}_{X} D$.

For the remainder of this section assume that $B$ satisfies both conditions $F_{\alpha^{+}}$and $E_{\alpha}$ for an infinite cardinal $\alpha$. A topological space $X$ is called homogeneous if given points $p, q \in X$ there is a continuous onto function $f: X \rightarrow X$ such that $f^{-1}$ is a continuous function and $f(p)=q$. For our topological space $X$, we are not only able to show that $X$ is not homogeneous but we can exhibit distinct points $p, q \in X$ for which the above statement fails (i.e. we exhibit why $X$ is not homogeneous).

4.5. TheOREM. There are $\alpha^{+}$-good points $p, q \in X$ such that $q$, but not $p$, has the property of being in the closure of a strongly discrete subspace of $X$.

Proof. The ultrafilter $\mathscr{F}$ on $B$ which is constructed in the proof of 3.6 has the properties required of $q$. The ultrafilter constructed in 3.5 (for any infinite $\eta$ ) has the properties required of $p$ by 4.3 .

It is easily shown that the set of $\alpha^{+}$-good, $\alpha^{+}$-OK points of $X$ is dense in $X$. It then follows that the set of $\alpha^{+}$-good ultrafilters is not a homogeneous space itself.

In [vM1], van Mill generalized Kunen's construction of $c$-OK points to construct $c$-OK sets. This construction is easily adapted to the present situation. We will indicate how to prove this result but we do not as yet have a use for it. One possible use is suggested in 4.7. A function $f$ from a space $K$ onto a space $Y$ is said to be irreducible if $f(F) \neq Y$ for each proper closed subset $F \subset K$. Call a set $K \subset X$ an $\alpha^{+}-\operatorname{good}\left(\alpha^{+}-O K\right)$ set if $\{b \in B: K \subset \bar{b}\}$ is an $\alpha^{+}-\operatorname{good}\left(\alpha^{+}-\mathrm{OK}\right)$ filter. 
4.6. THEOREM. If $Y$ is a compact space which is a continuous image of $U(\alpha)$, then there is an $\alpha^{+}$-good, $\alpha^{+}$-OK set $K \subset X$ which maps onto $Y$ by a continuous irreducible map.

Proof. Let $Y$ be a space as above. We will simply indicate the necessary modifications to the proofs of 3.2 and 3.4. Let $\left\{b_{\gamma}: \gamma \in \alpha\right\} \subset B$ be the cellular family as chosen in $\S 3$ and let $\eta=\omega$ be as in 3.2 and 3.4. We first redefine $\mathscr{F}_{0}$, changing it only slightly. Let $h \in \mathscr{S}_{1}$ be chosen as in 3.2 and choose $\left\{c_{n}\right.$ : $n \in \omega\} \subset B$ so that $\bar{c}_{n} \cap \bigcup\left\{\bar{b}_{\gamma}: \gamma \in \alpha\right\}=h^{\leftarrow}(n)$. Let $\mathscr{F}_{0}$ be the filter generated by $\left\{1-c_{n}: n \in \omega\right\} \cup\left\{b \in B:\left|\left\{\gamma<\alpha: b_{\gamma}<b\right\}\right|<\alpha\right\}$ (note that $\left|h^{\leftarrow}(n)\right|=\alpha$ for each $n \in \omega)$. For a filter $\mathscr{F} \subset B$ let $K_{\mathscr{F}}=\bigcap\{\bar{b}: b \in \mathscr{F}\}$. We require the following

FACT. There is a map $H$ from $K_{\mathscr{F}_{0}}$ onto $Y$.

To prove the Fact, it suffices to show that there is a map from $K_{\mathscr{F}_{0}}$ onto $U(\alpha)$. Let $e \in \alpha^{\alpha}$ be chosen so that, for each $n \in \omega,\left.e\right|_{h^{-}(n)}: h^{\leftarrow}(n) \rightarrow \alpha$ is an isomorphism. Define $g: \bigcup\left\{\bar{b}_{\gamma}: \gamma \in \alpha\right\} \rightarrow \alpha$ by $g(x)=e(\gamma)$ for $x \in \bar{b}_{\gamma}$. Since $B$ satisfies $F_{\alpha^{+}}, \cup\left\{\bar{b}_{\gamma}: \gamma \in \alpha\right\}$ is $C^{*}$-embedded in $X$. From this it follows that there is a continuous map

$$
\beta g: \operatorname{cl}_{X} \cup\left\{\bar{b}_{\gamma}: \gamma \in \alpha\right\} \rightarrow \beta \alpha
$$

such that $\left.\beta g\right|_{\cup\left\{\bar{b}_{\gamma}: \gamma \in \alpha\right\}}=g$. We show that the desired map $H$ is the restriction of $\beta g$ to $K_{\mathscr{F}_{0}}$. Since, for each $b \in \mathscr{F}_{0}$,

$$
\left|\alpha \backslash g\left[\bar{b} \cap \bigcup\left\{\bar{b}_{\gamma}: \gamma \in \alpha\right\}\right]\right|<\alpha, \quad U(\alpha) \subset H\left[K_{\mathscr{F}_{0}}\right] .
$$

On the other hand, since $B$ satisfies $F_{\alpha^{+}}$, for each $\delta<\alpha$, there is a $b \in \mathscr{F}_{0}$ such that $\left\{\gamma \in \alpha: \bar{b} \wedge \bar{b}_{\gamma} \neq \varnothing\right\}=\alpha-\delta$. Therefore, $H\left[K_{\mathscr{F}_{0}}\right]=U(\alpha)$ and the Fact is verified.

In proofs of 3.2 and 3.4 we replace condition (v) by $\left(\mathrm{v}^{\prime}\right)$ : if $d_{\beta} \in B$ for $\beta<2^{\alpha}$, then either $d_{\beta} \in \mathscr{F}_{\beta+1}$ or $H\left[K_{\mathscr{F}_{\beta}} \cap \bar{d}_{\beta}\right] \neq Y$. We must also alter the conditions 3.2(iii) and 3.4(iii) by adding the condition: for any finite sets $F, G$ with $F \subset \mathscr{S}_{\beta}$ and $G \subset \mathscr{T}_{\beta}$ and functions $s \in\left(\alpha^{+}\right)^{F}$ and $t \in \alpha^{G}$ with $\mathscr{G}$ being the filter $\{b \in B$ : $b_{\gamma}<b$ for all $\gamma \in\{\gamma: g(\gamma)=t(g)$ for $\left.g \in G\}\right\}$,

$$
H\left[K_{\mathscr{F}_{\beta}} \cap K_{\mathscr{G}} \cap \bigcap\left\{\bar{A}_{f, s(f)}: f \in F\right\}\right]=Y .
$$

The proof then proceeds in the obvious way just as in [vM1]. When the filter $\mathscr{F}_{2^{\alpha}}=\mathscr{F}$ is constructed, $K=K_{\mathscr{F}}$ is the required set. To see $\left.H\right|_{K}$ is irreducible, suppose that $L \subset K$ is a proper closed set. Then for some $\beta<2^{\alpha}, L \subset \bar{d}_{\beta}$, while $K \backslash \bar{d}_{\beta} \neq \varnothing$. Consequently, $d_{\beta} \notin \mathscr{F}_{\beta+1}$, since $K \subset K_{\mathscr{F}_{\beta+1}}$. Therefore by $\left(\mathrm{v}^{\prime}\right)$, $H\left[K_{\mathscr{F}_{\beta}} \cap \bar{d}_{\beta}\right] \neq Y$ from which it follows that $H[L] \neq Y$.

4.7. Remarks. The construction in 4.6 can be used to exhibit various distinct topological types with techniques similar to those in [vM1]. It may also be possible to settle the question of whether there can be $\alpha^{+}-\mathrm{OK}$ ultrafilters which are not $\alpha^{+}$-good. For example, one could try to embed a space $Y$ into $X$ as an $\alpha^{+}$-OK set where $Y$ does not have $\alpha^{+}$-good points itself.

For the special case $\alpha=\omega$, Kunen showed that if $p \in X$ is an $\omega_{1}-$ OK point then $p \notin \mathrm{cl}_{X} D$ for any $D \subset X \backslash\{p\}$ with $|D|<\omega_{1}$ (such a point is called a weak $P$-point). Kunen showed that $\omega^{*}$ has $c$-OK points, hence weak $P$-points, and from 
3.4 and 4.2 or 4.4 it follows that this is the case for $S(B)$ for any boolean algebra $B$ of cardinality $2^{\omega}$ and having properties $F_{\omega_{1}}$ and $E_{\omega}$ (more is shown in [vM1, vM2 and DvM].) One naturally wonders if these results generalize to larger $\alpha$, in particular what about $U(\alpha)$. However, the problems involved with larger cardinals as opposed to $\omega$ seem to be most evident in 4.4. For example, each countable discrete set is strongly discrete but this is not true for even discrete sets of cardinality $\omega_{1}$. However, by the following result provided by the referee, the remaining open problem is to find a point $p \in U(\alpha)$ satisfying $p \notin \operatorname{cl}(D)$ for any $D \subset U(\alpha) \backslash\{p\}$ of cardinality $\alpha$.

4.8. Proposition. If $p \in S(B)$ is $\alpha^{++}$-good then $p$ is not the limit point of any set $D$ of size at most $\alpha$.

Proof. By induction on $\alpha$ assume that $p$ is not a limit point of any set of size less than $\alpha$. Let $\left\{x_{\gamma}: \gamma<\alpha\right\} \subset S(B) \backslash\{p\}$. For $\beta<\alpha^{+}$, let $r_{\beta}$ be any injective map of $\beta$ into $\alpha$. Define $S$ from $\mathscr{P}_{\omega}\left(\alpha^{+}\right)$to be monotone so that, for $\gamma<\beta<\alpha^{+}$, $S(\{\gamma, \beta\})$ is a neighborhood of $p$ with $S(\{\gamma, \beta\}) \cap\left\{x_{\delta}: \delta<r_{\beta}(\gamma)\right\}=\varnothing$ (by the induction assumption). Assume that $I$ is the map from $\alpha^{+}$into the neighborhoods of $p$ given by $\alpha^{++}$-goodness. For each $\delta<\alpha$, let $Y_{\delta}=\left\{\beta<\alpha^{+}: x_{\delta} \in I(\beta)\right\}$. Each $Y_{\delta}$ has order type at most $\alpha$, for otherwise there are $\gamma<\beta$ in $Y_{\delta}$ with $r_{\beta}(\gamma)>\delta$ and so $x_{\delta} \in I(\gamma) \cap I(\beta)$ but $x_{\delta} \notin S(\{\gamma, \beta\})$. Therefore there is a $\gamma<\alpha^{+}$with $I(\gamma) \cap\left\{x_{\delta}\right.$ : $\delta<\alpha\}=\varnothing$.

\section{BIBLIOGRAPHY}

[BF] B. Balcar and F. Franek, Independent families in complete boolean algebras, Trans. Amer. Math. Soc. 274 (1982), 607-618.

[CN] W. W. Comfort and S. Negrepontis, The theory of ultrafilters, Springer-Verlag, New York, 1974.

[DvM] A. Dow and J. van Mill, On nowhere dense ccc P-sets, Proc. Amer. Math. Soc. 80 (1980), $697-700$

[EK] R. Engelking and M. Karlowicz, Some theorems of set theory and their topological consequences, Fund. Math. 57 (1965), 275-285.

[GJ] L. Gillman and M. Jerison, Rings of continuous functions, Van Nostrand, Princeton, N. J., 1960.

[Ke1] H. J. Keisler, Good ideals in fields of sets, Ann. of Math. (2) 79 (1964), 338-359.

[Ke2] __ Ideals with prescribed degree of goodness, Ann. of Math. (2) 81 (1965), 112-116.

[K1] K. Kunen, Ultrafilters and independent sets, Trans. Amer. Math. Soc. 172 (1972), 299-306.

[K2] Weak P-points in $\beta N-N$, Proc. Bolyai Janos Soc. Colloq. on Topology (Budapest), 1978, pp. 741-749.

[vM1] J. van Mill, Sixteen topological types in $\beta N-N$, Topology Appl. 13 (1981), 43-57.

[vM2] _. Weak P-points in Čech-Stone remainders, Trans. Amer. Math. Soc. (to appear).

[N1] S. Negrepontis, Extensions of continuous functions to $\beta D$, Indag. Math. 30 (1968), 393-400.

[N2] _ The Stone space of the saturated boolean algebras, Trans. Amer. Math. Soc. 141 (1969), $515-527$

[N3] $345-367$. , Adequate ultrafilters of special boolean algebras, Trans. Amer. Math. Soc. 174 (1972),

Department of Mathematics, University of Toronto, Toronto M5S 1A1, Ontario, Canada 